\title{
ON THE RELATIONSHIP BETWEEN AP, RS AND CEP
}

\author{
KEITH A. KEARNES
}

(Communicated by Donald Passman)

\begin{abstract}
We prove that in a residually small congruence modular variety the amalgamation property implies the commutator condition $\mathbf{R}$. A consequence of this is that, for all congruence modular varieties, AP+RS implies the CEP. We also show that this implication holds for any variety of semigroups.
\end{abstract}

\section{INTRODUCTION}

In W. Taylor's survey of equational logic [14] he states that, for varieties of algebras, the only possible relationship between the amalgamation property, residual smallness and the congruence extension property is the implication $\mathrm{AP}+\mathrm{RS} \Rightarrow \mathrm{CEP}$. Indeed, it is easy to find examples of congruence distributive varieties exhibiting any of the seven combinations of these properties other than the one this implication would exclude. Here is a table of examples where we have chosen the varieties to be finitely generated when possible

\begin{tabular}{|c|c|}
\hline Properties & Varieties \\
\hline $\mathrm{AP}+\mathrm{RS}+\mathrm{CEP}$ & Distributive Lattices \\
\hline $\mathrm{AP}+\mathrm{RS}+\neg \mathrm{CEP}$ & $?$ \\
\hline $\mathrm{AP}+\neg \mathrm{RS}+\mathrm{CEP}$ & Heyting Algebras \\
\hline $\mathrm{AP}+\neg \mathrm{RS}+\neg \mathrm{CEP}$ & Lattices $^{-}\left(\mathbf{F}_{4}, \mathbf{F}_{8}\right)$ \\
\hline$\neg \mathrm{AP}+\mathrm{RS}+\mathrm{CEP}$ & $\mathscr{V}\left(\mathbf{M}_{3}\right)$ \\
\hline$\neg \mathrm{AP}+\mathrm{RS}+\neg \mathrm{CEP}$ & Modular Lattices \\
\hline$\neg \mathrm{AP}+\neg \mathrm{RS}+\mathrm{CEP}$ & Cylindric Algebras (of a fixed dimension $)$ \\
\hline$\neg \mathrm{AP}+\neg \mathrm{RS}+\neg \mathrm{CEP}$ & \\
\hline
\end{tabular}

In this table, $\mathscr{V}\left(\mathbf{F}_{4}, \mathbf{F}_{8}\right)$ is the variety of commutative rings that is generated by the 4-element field and the 8-element field. $\mathscr{V}\left(\mathbf{M}_{3}\right)$ is the variety of lattices generated by the 5 -element simple lattice.

Until recently, there was no proof of the implication $A P+R S \Rightarrow C E P$, even for congruence distributive (CD) varieties. In fact, no progress was made on the problem until after Emil Kiss proved (in [9]) that for congruence modular

Received by the editors July 11,1988 .

1980 Mathematics Subject Classification (1985 Revision). Primary 08B10, 08B25. 
varieties, $\mathrm{CEP} \Rightarrow \mathrm{C} 2+\mathrm{R}$. Here, $\mathrm{C} 2$ and $\mathrm{R}$ are congruence conditions involving the modular commutator. They are

$$
\begin{aligned}
& C 2:[x, y]=x \cdot y \cdot[1.1], \text { and } \\
& \left.\mathbf{R}: \text { If } \mathbf{B} \leq \mathbf{A}, \quad \text { then }\left[1_{\mathbf{B}}, 1_{\mathbf{B}}\right]=\left[1_{\mathbf{A}}, 1_{\mathbf{A}}\right]\right]_{\mathbf{B}} .
\end{aligned}
$$

This suggested a possible program for proving $\mathrm{AP}+\mathrm{RS} \Rightarrow \mathrm{CEP}$ for congruence modular varieties. Three statements needed to be proved

Conjecture 1. $\mathrm{CM}+\mathrm{AP}+\mathrm{RS} \Rightarrow \mathrm{C} 2$,

Conjecture 2. $\mathrm{CM}+\mathrm{AP}+\mathrm{RS} \Rightarrow \mathrm{R}$, and

Conjecture 3. $\mathrm{CM}+\mathrm{AP}+\mathrm{RS}+\mathrm{C} 2+\mathrm{R} \Rightarrow \mathrm{CEP}$.

C. Bergman began this line of investigation with [1]. The principal result of that paper was that $\mathrm{CM}+\mathrm{AP}+\mathrm{RS}+\mathrm{C} 2+\mathrm{R}+\mathrm{F} 4 \Rightarrow \mathrm{CEP}$. Here, $\mathrm{F} 4$ is the condition that the free algebra on four generators in the variety $\mathscr{V}$ is finite. Each of the properties in this implication is insensitive to the addition of constants to the language of $\mathscr{V}$ except F4, which could be taken as a hint that a proof without finiteness assumptions (i.e., a proof of Conjecture 3 ) might be possible. Such a proof, and a proof of Conjecture 1, were discovered by R. McKenzie. The proofs of both of these results can be found in [3]. The purpose of the next section is to prove Conjecture 2. The main result of $\S 3$ is to show that $\mathrm{AP}+\mathrm{RS} \Rightarrow \mathrm{CEP}$ for all varieties of semigroups or monoids.

Our notation for universal algebra is fairly standard and the reader is referred to [12]. We will use some additional notation with respect to congruences and congruence lattices. We depart from [12] by using the symbols " + " and "." for the lattice operations "join" and "meet". If $f: \mathbf{B} \rightarrow \mathbf{A}$ is a homomorphism and $\theta$ is a congruence on $\mathbf{A}$, then we will use both $\left.\theta\right|_{\mathbf{B}}$ and $f^{-1}(\theta)$ to denote the congruence on $\mathbf{B}$ defined as $\{(x, y) \in \mathbf{B} \times \mathbf{B} \mid(f(x), f(y)) \in \theta\}$. If $\alpha$ is a congruence on $\mathbf{A}_{i}$ and $\mathbf{A}_{i}$ is the $i$ th factor in the product $\prod_{j \in I} \mathbf{A}_{j}$, then we will write $\alpha_{i}$ to denote the congruence $\pi_{i}^{-1}(\alpha)$ where $\pi_{i}$ is the canonical projection homomorphism of $\prod_{j \in I} \mathbf{A}_{j}$ onto its $i$ th coordinate. The only exception to this last rule is then $\alpha$ is the zero congruence of $\mathbf{A}_{i}$, in which case we write $\eta_{i}$ instead of $0_{i}$. We may abbreviate $\eta_{i} \cdot \eta_{j}$ by $\eta_{i \times j}$.

For the notation and basic results of modular commutator theory the reader is referred to [6].

A variety of algebras $\mathscr{V}$ is said to have the amalgamation property (AP) if whenever we have embeddings $f_{1}: \mathbf{A} \rightarrow \mathbf{B}$ and $g_{1}: \mathbf{A} \rightarrow \mathbf{C}$ we can find an algebra $\mathbf{D}$ and embeddings $f_{2}, g_{2}$ which complete a commutative diagram

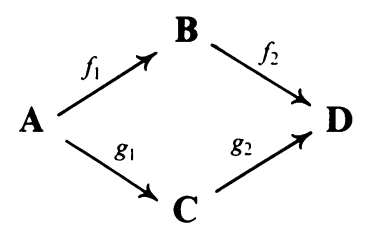

$\mathscr{V}$ is residually small (RS) just in case it has a bound on the size of its subdirectly irreducible members. $\mathscr{V}$ has the congruence extension property (CEP) 
only if for every embedding $f: \mathbf{B} \rightarrow \mathbf{A}$ and every congruence $\theta \in$ Con $\mathbf{B}$ there is a congruence $\psi \in \operatorname{Con} \mathbf{A}$ such that $\left.\psi\right|_{\mathbf{B}}=\theta$.

We will abbreviate the hypotheses that a variety $\mathscr{V}$ is congruence modular or that $\mathscr{V}$ has the amalgamation property or that every algebra in $\mathscr{V}$ satisfies the congruence conditions $\mathrm{C} 2$ and $\mathrm{R}$ etc. by writing " $\mathscr{V} \vDash \mathrm{CM}$ " or " $\mathscr{V} \vDash \mathrm{AP}$ " or " $\mathscr{V} \vDash \mathrm{C} 2+\mathrm{R}$ " etc.

\section{CONGRUENCE MODULAR VARIETIES}

Two concepts will be used throughout this section.

Definition 2.1. An algebra $\mathbf{A}$ is an essential extension of B (or $\mathbf{B}$ is essentially embedded in $\mathbf{A})$ if and only if $\mathbf{B}$ is embedded in $\mathbf{A}$ and for all $\theta \in \operatorname{Con} \mathbf{A}$ we have the property that $\left.\theta\right|_{\mathbf{B}}=0_{\mathbf{B}}$ implies that $\theta=0_{\mathbf{A}}$. We will write this as B $\leq_{\text {ess }}$ A.

Definition 2.2. A is finitely subdirectly irreducible if and only if for all pairs of congruences $\alpha, \beta \in \mathrm{Con} \mathbf{A}$ satisfying $\alpha \cdot \beta=0$ we must have $\alpha=0$ or $\beta=0$. We will write $\mathbf{A}^{F S I}$ to mean that $\mathbf{A}$ is a finitely subdirectly irreducible algebra. If $\mathbf{A}$ is also nonabelian, then we will write $\mathbf{A}^{\text {NAFSI }}$.

Prime algebras are those that satisfy the congruence condition that $[\alpha, \beta]=0$ if and only if $\alpha=0$ or $\beta=0$. Nontrivial prime algebras are always nonabelian and finitely subdirectly irreducible. In the presence of $\mathrm{C} 2$ the converse is true, the NAFSI algebras are precisely the nontrivial prime algebras.

We have the important result:

Theorem 2.3 (Taylor [13]). Every algebra in a residually small variety can be essentially embedded into another algebra which is in the variety and which is maximal with respect to $\leq_{\mathrm{ess}}$.

An element $x$ of a lattice $\mathbf{L}$ is said to be neutral if and only if for any two elements $y, z \in L$, the sublattice generated by $x, y$ and $z$ is distributive. In fact, in the presence of modularity, neutrality follows from the weaker assertion that $x \cdot(y+z)=x \cdot y+x \cdot z$ for all $y, z \in L$ (see Theorem 4 and Corollary 8 of $\S$ IIII.2 of [7]).

Lemma 2.4. Assume that $\mathscr{V} \vDash \mathrm{CM}+\mathrm{C} 2$ and that $\mathbf{A}_{0}, \mathbf{A}_{1} \in \mathscr{V}$. Then,

(a) $\theta \leq\left[1_{\mathbf{A}_{0}}, 1_{\mathbf{A}_{0}}\right]$ implies that $\theta$ is a neutral element of Con $\mathbf{A}_{0}$.

(b) $\theta \leq\left[1_{\mathbf{A}_{0} \times \mathbf{A}_{1}}, 1_{\mathbf{A}_{0} \times \mathbf{A}_{1}}\right]$ implies that $\theta$ is a product conruence on $\mathbf{A}_{0} \times \mathbf{A}_{1}$. Proof. For part (a), the additivity of the commutator along with two applications of $\mathrm{C} 2$ yields

$$
\begin{gathered}
\theta \cdot(\alpha+\beta)=\theta \cdot[1,1] \cdot(\alpha+\beta)=[\theta, \alpha+\beta]=[\theta, \alpha]+[\theta, \beta] \\
=\theta \cdot[1,1] \cdot \alpha+\theta \cdot[1,1] \cdot \beta=\theta \cdot \alpha+\theta \cdot \beta .
\end{gathered}
$$

By [7], $\theta$ is neutral.

In (b) we use the fact that $\theta$ is neutral to write $\theta=\left(\eta_{0}+\theta\right) \cdot\left(\eta_{1}+\theta\right)=\alpha_{0} \cdot \alpha_{1}^{\prime}$ for appropriate $\alpha \in \operatorname{Con} \mathbf{A}_{0}, \alpha^{\prime} \in \operatorname{Con} \mathbf{A}_{1}$. 
We begin a sequence of lemmas which culminate in the proof that, in the presence of $\mathrm{CM}+\mathrm{AP}+\mathrm{RS}, \mathbf{B} \leq_{\text {ess }} \mathbf{A}^{\mathrm{NAFSI}}$ implies that $\mathbf{B}$ is a NAFSI algebra. One may skip directly to the proof of Theorem 2 if one assumes this fact.

Lemma 2.5. Let $\mathscr{V} \vDash \mathrm{CM}+\mathrm{C} 2$. Assume that $\mathbf{B}_{0} \leq_{\text {ess }} \mathbf{A}_{0}^{\text {NAFSI }}$ and that $\mathbf{B}_{1} \leq_{\text {ess }}$ $\mathbf{A}_{1}$. Then $\mathbf{B}_{0} \times \mathbf{B}_{1} \leq_{\text {ess }} \mathbf{A}_{0} \times \mathbf{A}_{1}$. In particular,

(a) $\mathbf{B} \leq_{\text {ess }} \mathbf{A}^{N A F S I} \Rightarrow \mathbf{B} \times \mathbf{B} \leq_{\text {ess }} \mathbf{A} \times \mathbf{A}$ and,

(b) $\mathbf{B} \leq_{\text {ess }} \mathbf{A}^{N A F S I} \Rightarrow \mathbf{B} \times \mathbf{C} \leq_{\text {ess }} \mathbf{A} \times \mathbf{C}$.

Proof. Suppose $\psi \in \operatorname{Con} \mathbf{A}_{0} \times \mathbf{A}_{1}$ satisfies $\left.\psi\right|_{\mathbf{B}_{0} \times \mathbf{B}_{1}}=0$. Since $\psi \cdot[1,1]$ is a product congruence on $\mathbf{A}_{0} \times \mathbf{A}_{1}$ and $\left.\psi \cdot[1,1]\right|_{\mathbf{B}_{0} \times \mathbf{B}_{1}}=0$ we must have $\psi \cdot[1,1]=$ 0 . By the neutrality of $[1,1]$, this means that $\eta_{0}=\left(\eta_{0}+\psi\right) \cdot\left(\eta_{0}+[1,1]\right)$. However, $\mathbf{A}_{0}$ is a NAFSI algebra. Since $\mathbf{A}_{0}$ is nonabelian, $\eta_{0}<\eta_{0}+[1,1]$. $\mathbf{A}_{0}$ is FSI, so we are forced to conclude that $\eta_{0}=\eta_{0}+\psi$. This shows that $\psi \leq \eta_{0}$, so $\psi$ must be of the form $\eta_{0} \cdot \alpha_{1}$ for some congruence $\alpha \in \operatorname{Con} \mathbf{A}_{1}$ that satisfies $\left.\alpha\right|_{\mathbf{B}_{1}}=0$. But $\mathbf{B}_{1} \leq_{\text {ess }} \mathbf{A}_{1}$ so the only suitable choice is $\alpha=0$. Therefore $\psi=\eta_{0} \cdot \eta_{1}=0$. The special cases (a) and (b) are direct consequences of the Lemma.

Lemma 2.6. Assume that $\mathscr{V} \vDash C M+C 2$, and that $\mathbf{A}^{N A F S I}$ and $\mathbf{C}$ are members of $\mathscr{V}$ such that $\mathbf{A}$ is maximal with respect to $\leq_{\mathrm{ess}}$ and $\mathbf{A} \times \mathbf{A} \leq_{\mathrm{ess}} \mathbf{C}$. Then there exists $\psi \in$ Con $\mathbf{C}$ such that $\left.\psi\right|_{\mathbf{A} \times \mathbf{A}}=\eta_{0}^{\mathbf{A} \times \mathbf{A}}$ or $\eta_{1}^{\mathbf{A} \times \mathbf{A}}$.

Proof. Embed $\mathbf{A}$ into $\mathbf{A} \times \mathbf{A}$ via the diagonal homomorphism, $\delta$. Choose $\psi \in$ Con $\mathbf{C}$ maximal with respect to the property that $\left.\psi\right|_{\mathbf{A}}=0$. Because of the maximality condition on $\psi$, the induced homomorphism from $\mathbf{A}$ to $\mathbf{C} / \psi$ given by the composition

$$
\mathbf{A} \stackrel{\delta}{\longrightarrow} \mathbf{A} \times \mathbf{A} \hookrightarrow \mathbf{C} \stackrel{\text { nat }}{\longrightarrow} \mathbf{C} / \psi
$$

is an essential embedding. The hypothesis on $\mathbf{A}$ implies that this embedding is an isomorphism. Hence, $\mathbf{A} \times \mathbf{A} /\left.\psi\right|_{\mathbf{A} \times \mathbf{A}} \cong \mathbf{A}$. Writing $\widetilde{\psi}$ for $\left.\psi\right|_{\mathbf{A} \times \mathbf{A}}$, this shows that $\widetilde{\psi}$ is a meet irreducible element of $\operatorname{Con} \mathbf{A} \times \mathbf{A}$ that is not above $[1,1]$. But $\left(\widetilde{\psi}+\eta_{0}\right) \cdot\left(\left(\widetilde{\psi}+\eta_{1}\right) \cdot(\widetilde{\psi}+[1,1])\right)=\left(\widetilde{\psi}+\eta_{0}\right) \cdot\left(\widetilde{\psi}+\eta_{1} \cdot[1,1]\right)=\widetilde{\psi}$, so for some $i=0$ or 1 we must have $\widetilde{\psi}=\widetilde{\psi}+\eta_{i}$, i.e. that $\widetilde{\psi} \geq \eta_{i}$. If $\psi>\eta_{i}$ then $\left.\psi\right|_{\mathbf{A}}=\left.\widetilde{\psi}\right|_{\mathbf{A}}>0$, which is false, so $\widetilde{\psi}=\eta_{i}$.

Lemma 2.7. Assume that $\mathscr{V} \vDash \mathrm{CM}+\mathrm{AP}+\mathrm{RS}$. If $\mathbf{B} \leq_{\text {ess }} \mathbf{A}^{\text {NAFSI }}$ where $\mathbf{A} \in$ $\mathscr{V}$, then $\mathbf{B}$ is nonabelian.

Proof. If $\mathbf{E}$ is an essential extension of a NAFSI algebra $\mathbf{F}$, then $\mathbf{E}$ is a NAFSI algebra. The verification that $\mathbf{E}$ must be a NAFSI algebra is as follows. First $\mathbf{E}$ must be nonabelian because it possesses a nonabelian subalgebra (e.g. F). Second, if there are nonzero congruences $\alpha$ and $\beta$ on $\mathbf{E}$ which intersect to the zero congruence, then $\left.\alpha\right|_{F}$ and $\left.\beta\right|_{F}$ are nonzero congruences on $\mathbf{F}$ which intersect to the zero congruence, contradicting the assumption that $\mathbf{F}$ is FSI. 
By the previous observation, and referring to Theorem 2.3, we see that we may assume that $\mathbf{A}$ is maximal with respect to $\leq_{\text {ess }}$. We may also assume that $\mathscr{V} \vDash \mathrm{C} 2$ by Theorem 1 of [3].

Assume that $\mathbf{B}$ is abelian and we will argue to a contradiction. Let $\mathbf{B}_{\nabla}$ be the linearization of $\mathbf{B}$, i.e. $\mathbf{B}_{\nabla}=(\mathbf{B} \times \mathbf{B}) / \Delta_{1,1}$ where $\Delta_{1,1}$ is the congruence on $\mathbf{B} \times \mathbf{B}$ generated by the set of all pairs $((x, x),(y, y))$ for $x, y \in \mathbf{B}$. According to Proposition 9.8 of [6], since $\mathscr{V}$ is congruence modular and $\mathbf{B}$ is abelian, the maps $\lambda_{0}: \mathbf{B} \times \mathbf{B} \rightarrow \mathbf{B}_{\nabla} \times \mathbf{B}:(x, y) \mapsto((x, y) / \Delta, y)$ and $\lambda_{1}: \mathbf{B} \times \mathbf{B} \rightarrow$ $\mathbf{B} \times \mathbf{B}_{\nabla}:(x, y) \mapsto(x,(x, y) / \Delta)$ are isomorphisms. Let $i$ be the embedding of $\mathbf{B}$ into $\mathbf{A}$ that is given. Let $h_{0}$ be the map from $\mathbf{B} \times \mathbf{B}$ to $\mathbf{B}_{\nabla} \times \mathbf{A}$ that is the composition $\left(\mathrm{id}_{\mathbf{B}_{\nabla}} \times i\right) \circ \lambda_{0}$. Similarly, let $h_{1}=\left(i \times \mathrm{id}_{\mathbf{B}_{\nabla}}\right) \circ \lambda_{1}$. By Lemma 2.5 the maps $i \times i, h_{0}$ and $h_{1}$ are all essential embeddings. Find an algebra $\mathbf{C}$ and embeddings which complete the following diagram

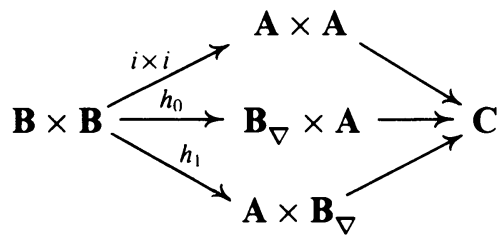

Choose $\xi \in$ Con $\mathbf{C}$ maximal with respect to the condition that $\left.\xi\right|_{\mathbf{B} \times \mathbf{B}}=0$. We can replace $\mathbf{C}$ by $\mathbf{C} / \xi=\mathbf{D}$ and complete the previous diagram in such a way that all maps are essential embeddings.

By Lemma 2.6 there is a $\psi \in$ Con $\mathbf{D}$ such that $\left.\psi\right|_{\mathbf{A} \times \mathbf{A}}=\eta_{0}^{\mathbf{A} \times \mathbf{A}}$ or $\eta_{1}^{\mathbf{A} \times \mathbf{A}}$, say $\left.\psi\right|_{\mathbf{A} \times \mathbf{A}}=\eta_{0}^{\mathbf{A} \times \mathbf{A}}$ (the other case is similar). Let $\widetilde{\psi}=\left.\psi\right|_{\mathbf{B}_{\nabla} \times \mathbf{A}}$. Then $\left.\widetilde{\psi}\right|_{\mathbf{B} \times \mathbf{B}}=$ $\left.\eta_{0}^{\mathbf{A} \times \mathbf{A}}\right|_{\mathbf{B} \times \mathbf{B}}=\eta_{0}^{\mathbf{B} \times \mathbf{B}},\left.\eta_{0}^{\mathbf{B} \nabla \times \mathbf{A}}\right|_{\mathbf{B} \times \mathbf{B}}=\Delta_{1,1},\left.\eta_{1}^{\mathbf{B} \nabla \times \mathbf{A}}\right|_{\mathbf{B} \times \mathbf{B}}=\eta_{1}^{\mathbf{B} \times \mathbf{B}}$, so for $j=0$ and for $j=1$ we have $h_{0}^{-1}\left(\widetilde{\psi} \cdot \eta_{j}^{\mathbf{B} \nabla \times \mathbf{A}}\right)=0_{\mathbf{B} \times \mathbf{B}}$. Since $h_{0}$ is essential, $\widetilde{\psi} \cdot \eta_{j}^{\mathbf{B}_{\nabla} \times \mathbf{A}}=$ $0_{\mathbf{B}_{\nabla \times \mathbf{A}}}$ for each $j$. A fortiori we have that the product congruences $(\widetilde{\psi} \cdot[1,1]) \cdot \eta_{j}$ are zero, so $\widetilde{\psi} \cdot[1,1]=0$ by Lemma 2.4 . Using the neutrality of $[1,1]$ we find that $\eta_{1}=\left(\eta_{1}+\widetilde{\psi}\right) \cdot\left(\eta_{1}+[1,1]\right)$. But $\eta_{1}$ is a meet-irreducible congruence of $\mathbf{B}_{\nabla} \times \mathbf{A}$ that is not above $[1,1]$, since $\mathbf{B}_{\nabla} \times \mathbf{A} / \eta_{1} \cong \mathbf{A}$. Hence, $\widetilde{\psi} \leq \eta_{1}$. Restricting these two congruences to $\mathbf{B} \times \mathbf{B}$ we learn that $\eta_{0}^{\mathbf{B} \times \mathbf{B}} \leq \eta_{1}^{\mathbf{B} \times \mathbf{B}}$, forcing the conclusion that $\mathbf{B}$ is trivial. This is a contradiction of our hypothesis that $\mathbf{B} \leq_{\text {ess }} \mathbf{A}^{\text {NAFSI }}$, for trivial algebras can only have trivial (hence abelian) essential extensions.

Lemma 2.8. Assume that $\mathscr{V} \vDash \mathrm{CM}+\mathrm{AP}+\mathrm{RS}$. If $\mathbf{B}_{0} \times \mathbf{B}_{1} \leq_{\mathrm{ess}} \mathbf{A}^{\text {NAFSI }}$ then either $\mathbf{B}_{0}$ or $\mathbf{B}_{1}$ is trivial.

Proof. We may assume that $\mathbf{A}$ is maximal with respect to $\leq_{\text {ess }}$. Let $i$ denote the embedding of $\mathbf{B}_{0} \times \mathbf{B}_{1}$ into $\mathbf{A}$ that is given. Also, let $\widehat{\mathbf{A}}$ denote a copy of A that we do not want to confuse with the original. 
Find an algebra $\mathbf{D}$ and embeddings to complete the diagram

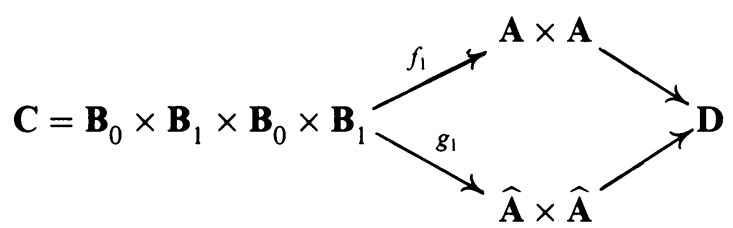

Here $f_{1}(a, b, c, d)=(i(a, b), i(c, d))$ and $g_{1}(a, b, c, d)=(i(a, d)$, $i(c, b))$. By Lemma 2.5(a) these maps are essential embeddings.

Choose $\xi \in$ Con $\mathbf{D}$ maximal with respect to the condition that $\left.\xi\right|_{\mathbf{C}}=0_{\mathbf{C}}$. We get a diagram

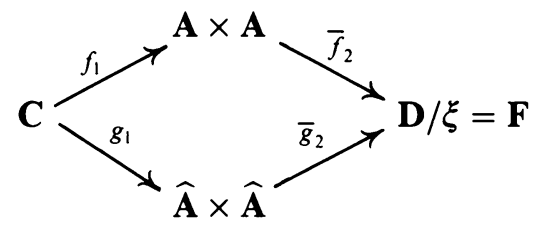

where all maps are essential embeddings. Use Lemma 2.6 to find a $\psi \in$ Con $\mathbf{F}$ such that $\bar{g}_{2}^{-1}(\psi)=\eta_{0}^{\widehat{\mathbf{A}} \times \widehat{\mathbf{A}}}$ or $\eta_{1}^{\widehat{\mathbf{A}} \times \widehat{\mathbf{A}}}$. The argument is similar for either case so say that $\bar{g}_{2}^{-1}(\psi)=\eta_{0}^{\widehat{\mathbf{A}} \times \widehat{\mathbf{A}}}$. Let $\widetilde{\psi}=\bar{f}_{2}^{-1}(\psi)$ and set $\beta=\eta_{0}^{\mathbf{A} \times \mathbf{A}}+\widetilde{\psi} \cdot \eta_{1}^{\mathbf{A} \times \mathbf{A}}$.

Claim. $f_{1}^{-1}(\beta)=\eta_{0}^{\mathbf{C}}$.

Proof of Claim. Clearly, $f_{1}^{-1}(\beta) \supseteq f_{1}^{-1}\left(\eta_{0}^{\mathbf{A} \times \mathbf{A}}\right)+f_{1}^{-1}\left(\widetilde{\psi} \cdot \eta_{1}^{\mathbf{A} \times \mathbf{A}}\right)=\eta_{0 \times 1}^{\mathbf{C}}+\eta_{0 \times 2 \times 3}^{\mathbf{C}}=$ $\eta_{0}^{\mathrm{C}}$, so we only need to show that $f_{1}^{-1}(\beta) \subseteq \eta_{0}^{\mathrm{C}}$. Let $(x, y)$ be an arbitrary element of $f_{1}^{-1}(\beta)$. Write the coordinates as $x=(a, b, c, d), y=$ $\left(a^{\prime}, b^{\prime}, c^{\prime}, d^{\prime}\right) \in \mathbf{C}=\mathbf{B}_{0} \times \mathbf{B}_{1} \times \mathbf{B}_{0} \times \mathbf{B}_{1}$. Then $\left(f_{1}(x), f_{1}(y)\right) \in \eta_{0}+\widetilde{\psi} \cdot \eta_{1}$. Since $\eta_{0}$ permutes with $\tilde{\psi} \cdot \eta_{1}$ (see Exercise 5.4 of [6]), there is a pair $(u, v) \in \mathbf{A} \times \mathbf{A}$ such that $f_{1}(x)=(i(a, b), i(c, d)) \eta_{0}(u, v) \widetilde{\psi} \cdot \eta_{1}\left(i\left(a^{\prime}, b^{\prime}\right), i\left(c^{\prime}, d^{\prime}\right)\right)=f_{1}(y)$. Hence $(u, v)=\left(i(a, b), i\left(c^{\prime}, d^{\prime}\right)\right) \in \operatorname{im} f_{1}$, implying that $(x, y) \in f_{1}^{-1}\left(\eta_{0}\right)+$ $f_{1}^{-1}\left(\tilde{\psi} \cdot \eta_{1}\right)=\eta_{0}^{\mathrm{C}} \cdot \triangleleft$.

By similar arguments we can let $\gamma=\eta_{1}^{\mathbf{A} \times \mathbf{A}}+\tilde{\psi} \cdot \eta_{0}^{\mathbf{A} \times \mathbf{A}}$ and get that $f_{1}^{-1}(\gamma)=$ $\eta_{3}^{\mathrm{C}}$. Let $\alpha$ denote the congruence on $\mathbf{A}$ that corresponds to the congruence $\beta / \eta_{0}$ on $(\mathbf{A} \times \mathbf{A}) / \eta_{0}$ under the natural isomorphism $(\mathbf{A} \times \mathbf{A}) / \eta_{0} \stackrel{\sim}{\rightarrow} \mathbf{A}$. Similarly, let $\alpha^{\prime}$ denote the congruence on $\mathbf{A}$ that corresponds to the congruence $\gamma / \eta_{1}$ on $(\mathbf{A} \times \mathbf{A}) / \eta_{1}$. From what we have said about $\beta$ and $\gamma$ we see that $i^{-1}(\alpha)=$ $\eta_{0}^{\mathbf{B}_{0} \times \mathbf{B}_{1}}$ and $i^{-1}\left(\alpha^{\prime}\right)=\eta_{1}^{\mathbf{B}_{0} \times \mathbf{B}_{1}}$, so $i^{-1}\left(\alpha \cdot \alpha^{\prime}\right)=0_{\mathbf{B}_{0} \times \mathbf{B}_{1}}$. Since $i$ is essential, $\alpha \cdot \alpha^{\prime}=0_{\mathbf{A}}$. $\mathbf{A}$ is finitely subdirectly irreducible so either $\alpha$ or $\alpha^{\prime}$ equals $0_{\mathbf{A}}$. Both of these cases are similar so let's assume that $\alpha=0_{\mathbf{A}}$. Then, $0_{\mathbf{B}_{0} \times \mathbf{B}_{1}}=$ $i^{-1}(\alpha)=\eta_{0}^{\mathbf{B}_{0} \times \mathbf{B}_{1}}$ and the conclusion is that $\mathbf{B}_{1}$ is trivial.

Lemma 2.9. Assume that $\mathscr{V} \vDash \mathrm{CM}+\mathrm{AP}+\mathrm{RS}$. If $\mathbf{B} \leq_{\mathrm{ess}} \mathbf{A}^{\text {NAFSI }}$ then $\mathbf{B}$ is a NAFSI algebra. 
Proof. By Lemma 2.7, B is nonabelian. Assume that $\mathbf{B}$ is finitely subdirectly reducible. Then there exist $\alpha, \beta>0$ in Con B such that $\alpha \cdot \beta=0$. We may assume that $\alpha \leq[1,1]$, for, if $\alpha \cdot[1,1] \neq 0$, then we may replace $\alpha$ by $\alpha \cdot[1,1]$. On the other hand, if $\alpha \cdot[1,1]=0$, then we may replace $\alpha$ by $[1,1]$ and replace $\beta$ by the old choice for $\alpha$. Now we may assume that $\beta$ is equal to $(0: \alpha)$, the centralizer of $\alpha$. This is because $0=\alpha \cdot \beta$ iff $0=[1,1] \cdot \alpha \cdot \beta=[\alpha, \beta]$ iff $\beta \leq(0: \alpha)$. Hence, if there is some congruence $\beta$ satisfying $\beta>0$ and $\alpha \cdot \beta=0$, then $(0: \alpha)$ is another (in fact, the largest) such congruence.

Find an algebra $\mathbf{C}$ and embeddings that complete the following diagram

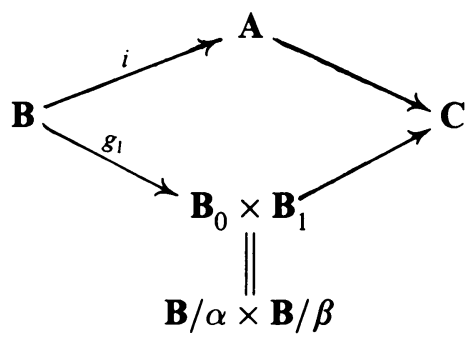

The map $g_{1}$ is the homomorphism that is the natural map in both coordinates. The map $i$ is the embedding of $\mathbf{B}$ into $\mathbf{A}$ that is given.

Choose $\xi \in$ Con $\mathbf{C}$ maximal with respect to the condition that $\left.\xi\right|_{\mathbf{B}}=0_{\mathbf{B}}$. Let $\tilde{\xi}=\left.\xi\right|_{\mathbf{B}_{0} \times \mathbf{B}_{1}}$. In $\operatorname{Con} \mathbf{B}_{0} \times \mathbf{B}_{1}, \tilde{\xi} \cdot[1,1]$ is a product congruence. Say that $\tilde{\xi} \cdot[1,1]=\gamma_{0} \cdot \gamma_{1}^{\prime}$ where $\gamma \in \operatorname{Con} \mathbf{B}_{0}$ and $\gamma^{\prime} \in \operatorname{Con} \mathbf{B}_{1}$. Pulling these congruences back to $\mathbf{B}$ we get $\left.\gamma_{0}\right|_{\mathbf{B}}=\tilde{\gamma} \geq \alpha$ and $\left.\gamma_{1}^{\prime}\right|_{\mathbf{B}}=\tilde{\gamma}^{\prime} \geq \beta$. Now, $\alpha \cdot \tilde{\gamma}^{\prime} \leq \tilde{\gamma} \cdot \tilde{\gamma}^{\prime}=0$ so $\tilde{\gamma}^{\prime} \leq(0: \alpha)=\beta$. Thus, $\tilde{\gamma}^{\prime}=\beta$. In Con $\mathbf{B}_{0} \times \mathbf{B}_{1}$ we get that $\xi \cdot[1,1] \leq \gamma_{1}^{\prime}=\eta_{1}$, so we can use the neutrality of $[1,1]$ to deduce that $\eta_{1}=\left(\eta_{1}+\widetilde{\xi}\right) \cdot\left(\eta_{1}+[1,1]\right)$. However, $\alpha \cdot\left(\left.\eta_{1}\right|_{\mathbf{B}}\right)=\alpha \cdot \beta=0$ and $\left.\alpha \cdot\left(\eta_{1}+[1,1]\right)\right|_{\mathbf{B}} \geq \alpha \cdot\left[1_{\mathbf{B}}, 1_{\mathbf{B}}\right]=\alpha$, so necessarily $\left.\alpha \cdot\left(\eta_{1}+\widetilde{\xi}\right)\right|_{\mathbf{B}}=0$. This gives us a sandwich: $\beta=\left.\eta_{1}\right|_{\mathbf{B}} \leq\left.\left(\eta_{1}+\tilde{\xi}\right)\right|_{\mathbf{B}} \leq(0: \alpha)=\beta$ which forces $\eta_{1}+\widetilde{\xi}=\eta_{1}$. Hence, $\widetilde{\xi}=\tau_{0} \cdot \eta_{1}$ for some $\tau \in \operatorname{Con} \mathbf{B}_{0}$.

We get a commutative diagram in which all maps are embeddings

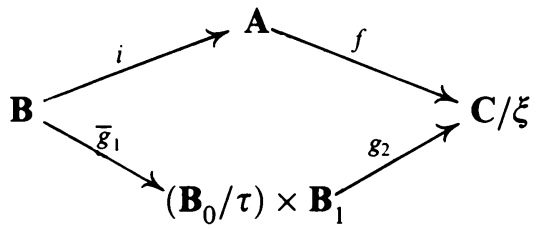

The map $i$ is essential by hypothesis, $f$ is essential because of the choice of $\xi$ and $g_{2}$ is essential because $g_{2} \circ \bar{g}_{1}=f \circ i$ is. Since A is a NAFSI algebra and $f$ is essential, $\mathbf{C} / \xi$ is a NAFSI. By Lemma 2.8 , either $\mathbf{B}_{0} / \tau$ or $\mathbf{B}_{1}$ is trivial. But then the natural mapping of $\mathbf{B}$ onto either $\mathbf{B}_{1}=\mathbf{B} / \beta$ or $\mathbf{B}_{0} / \tau \cong \mathbf{B} /\left(\alpha+\left.\xi\right|_{\mathbf{B}}\right)$ is an embedding. This leads to the contradication that either $\beta$ or $\alpha=0$.

We can now prove the main result of this section. 
Theorem 2.10. Assume that $\mathscr{V} \vDash \mathrm{CM}+\mathrm{AP}+\mathrm{RS}$. Then $\mathscr{V} \vDash R$.

Proof. Assume not. Then there exists $\mathbf{A} \in \mathscr{V}$ and a subalgebra $\mathbf{B} \leq \mathbf{A}$ such that $\left.\left[1_{\mathbf{A}}, 1_{\mathbf{A}}\right]\right|_{\mathbf{B}}>\left[1_{\mathbf{B}}, 1_{\mathbf{B}}\right]$. We will use the abbreviations $\alpha=\left.\left[1_{\mathbf{A}}, 1_{\mathbf{A}}\right]\right|_{\mathbf{B}}$ and $\beta=\left[1_{\mathbf{B}}, 1_{\mathbf{B}}\right]$ in this proof. Let $\Delta_{1, \alpha}$ denote the congruence on $\mathbf{B} \times \mathbf{B}$ generated by all the pairs $((x, x),(y, y))$ for all $x$ and $y$ for which $(x, y) \in \alpha$. Let $\mathbf{C}$ denote the (abelian) algebra $\mathbf{B} /\left[1_{\mathbf{B}}, 1_{\mathbf{B}}\right]$. In Con $\mathbf{B} \times \mathbf{C}$ we have the following sublattice

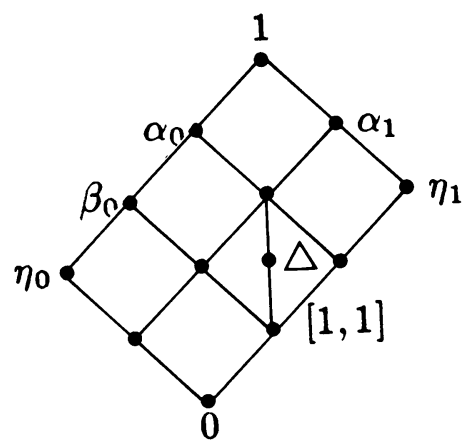

The congruence $\Delta$ is the projection of the congruence $\Delta_{1, \alpha} \in \operatorname{Con} \mathbf{B} \times \mathbf{B}$ onto $\mathbf{B} \times \mathbf{C}$. That is, $\Delta$ corresponds to $\Delta_{1, \alpha} /\left(\eta_{0} \cdot \beta_{1}\right)$ under the natural identification of $(\mathbf{B} \times \mathbf{B}) /\left(\eta_{0} \cdot \beta_{1}\right)$ with $\mathbf{B} \times \mathbf{C}$.

The only mystery in the previous diagram is the placement of $\Delta$. In Con $\mathbf{B} \times \mathbf{B}$ we have $\eta_{j} \cdot \Delta_{1, \alpha}=\eta_{j} \cdot[1, \alpha]_{1-j}=\eta_{j} \cdot \beta_{1-j}$ by $C 2$. Further, arguing by generators we see that $\eta_{j}+\Delta_{1, \alpha}=\alpha_{j}$. Using modularity this is already enough to show that $\Delta_{1, \alpha}$ is a common complement to $\alpha_{0} \cdot \beta_{1}$ and $\alpha_{1} \cdot \beta_{0}$ in the interval $I\left[\beta_{0} \cdot \beta_{1}, \alpha_{0} \cdot \alpha_{1}\right]$ of $\operatorname{Con} \mathbf{B} \times \mathbf{B}$ (or that $\Delta$ is a common complement to $\alpha_{0} \cdot \eta_{1}$ and $\alpha_{1} \cdot \beta_{0}$ in the interval $I\left[\beta_{0} \cdot \eta_{1}, \alpha_{0} \cdot \alpha_{1}\right]$ of $\left.\operatorname{Con} \mathbf{B} \times \mathbf{C}\right)$.

Let $\mathbf{D}$ denote the (abelian) algebra $(\mathbf{B} \times \mathbf{C}) / \Delta$ and let $i$ denote the inclusion of $\mathbf{B}$ into $\mathbf{A}$. Find an algebra $\mathbf{F}$ and embeddings that complete the following diagram:

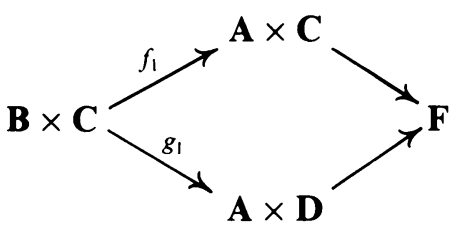

Here $f_{1}=i \times \mathrm{id}_{\mathrm{C}}$ and $g_{1}(b, c)=(i(b),(b, c) / \Delta) . g_{1}$ is an embedding since $\operatorname{ker} g_{1}=\eta_{0} \cdot \Delta=0$.

Choose $(c, d) \in \eta_{0} \cdot \alpha_{1}-0_{\mathbf{B} \times \mathbf{C}}$ and find $\tau \in$ Con $\mathbf{F}$ maximal with respect to the condition that $\left.(c, d) \notin \tau\right|_{\mathbf{B} \times \mathbf{C}}+\left[1_{\mathbf{B} \times \mathbf{C}}, 1_{\mathbf{B} \times \mathbf{C}}\right]$. This is possible since $(c, d) \notin[1,1]$ (which follows from the fact that $\eta_{0} \cdot \alpha_{1} \cdot[1,1]=0$ ). Now, $\left.\left[1_{\mathbf{F}}, 1_{\mathbf{F}}\right]\right|_{\mathbf{B} \times \mathbf{C}} \geq\left.\left[1_{\mathbf{A} \times \mathbf{C}}, 1_{\mathbf{A} \times \mathbf{C}}\right]\right|_{\mathbf{B} \times \mathbf{C}}+\left.\left[1_{\mathbf{A} \times \mathbf{D}}, 1_{\mathbf{A} \times \mathbf{D}}\right]\right|_{\mathbf{B} \times \mathbf{C}}=\alpha_{0} \cdot \eta_{1}+\alpha_{0} \cdot \Delta=\alpha_{0}$. $\alpha_{1} \supseteq \eta_{0} \cdot \alpha_{1}$. Since $(c, d) \in \eta_{0} \cdot \alpha_{1}$ we conclude that $\tau \geq\left[1_{\mathbf{F}}, 1_{\mathbf{F}}\right]$. Further, 
$\tau$ is meet-irreducible, since if $\gamma, \gamma^{\prime}>\tau$ then $(c, d)$ is an element of both $\left.\gamma\right|_{\mathbf{B} \times \mathbf{C}}+[1,1]$ and $\left.\gamma^{\prime}\right|_{\mathbf{B} \times \mathbf{C}}[1,1]$ by the maximality condition on $\tau$. Therefore, using the neutrality of $[1,1],(c, d) \in\left(\left.\gamma\right|_{\mathbf{B} \times \mathbf{C}}+[1,1]\right) \cdot\left(\left.\gamma^{\prime}\right|_{\mathbf{B} \times \mathbf{C}}+[1,1]\right)=$ $\left.\gamma \cdot \gamma^{\prime}\right|_{\mathbf{B} \times \mathbf{C}}+[1,1]$ which shows that $\gamma \cdot \gamma^{\prime}>\tau$. Thus, $\mathbf{F} / \tau$ is a NAFSI algebra. If we let $\tilde{\tau}=\left.\tau\right|_{\mathbf{B} \times \mathbf{C}}$ then we have an induced embedding:

$$
(\mathbf{B} \times \mathbf{C}) / \tilde{\tau} \leq_{\text {ess }}(\mathbf{F} / \tau)^{\text {NAFSI }} .
$$

This embedding is essential because of the maximality condition on $\tau$. By Lemma 2.9 , this means that $(\mathbf{B} \times \mathbf{C}) / \tilde{\tau}$ is a NAFSI algebra, i.e. that $\tilde{\tau}$ is a meet-irreducible congruence of $\mathbf{B} \times \mathbf{C}$ that is not above [1,1]. However, $\tilde{\tau}=(\tilde{\tau}+[1,1]) \cdot\left(\tilde{\tau}+\eta_{0} \cdot \alpha_{1}\right)$, forcing $\tilde{\tau}=\tilde{\tau}+\eta_{0} \cdot \alpha_{1}$. This proves the final contradiction that $(c, d) \in \eta_{0} \cdot \alpha_{1} \subseteq \tilde{\tau} \subseteq \tau$.

Corollary 2.11. Let $\mathscr{V}$ be a congruence modular variety. Then $\mathscr{V}$ satisfies the implication $\mathrm{AP}+\mathrm{RS} \Rightarrow \mathrm{CEP}$.

\section{VARIETIES OF SEMIGROUPS}

Congruence modular varieties and varieties of semigroups are at opposite ends of the spectrum determined by congruence conditions. On the other hand, varieties of semigroups are perhaps the most studied examples of varieties that are not congruence modular. For this reason, varieties of semigroups seem a good place to test the implication $\mathrm{AP}+\mathrm{RS} \Rightarrow \mathrm{CEP}$. Fortunately for us, researchers have already completely determined which varieties of semigroups are residually small [11] and which have the congruence extension property [5]. It is not likely that the varieties of semigroups with the amalgamation property will be completely determined soon, but we can still test the implication $\mathrm{AP}+\mathrm{RS} \Rightarrow \mathrm{CEP}$.

In what follows, we will call a semigroup in which every element has a twosided inverse with respect to some two-sided identity element a group. A semigroup homomorphism between two groups must preserve idempotents, hence it preserves the (unique) identity element and therefore inverses.

Lemma 3.1. Let $\mathscr{S}$ be a variety of semigroups which has AP. The subclass of groups $\mathscr{G} \subseteq \mathscr{S}$ also has AP.

Proof. Trivial. Any diagram of groups can be completed with embeddings into some semigroup, but the images of these embeddings generate a subgroup of this semigroup.

Indeed, it is easy to see that this theorem is true for more general categories of semigroups.

Corollary 3.2. If $\mathscr{S}$ is a variety of semigroups satisfying $\mathrm{AP}+\mathrm{RS}$, then $\mathscr{G}$ is a subvariety satisfying AP + RS.

Proof. Every residually small variety of semigroups satisfies $x^{n} \approx x^{2 n}$ for some $n$ by [11]. Therefore, if we add $x^{n} \cdot y \approx y$ and $y \cdot x^{n} \approx y$ to the laws of $\mathscr{S}$ 
we slice out exactly $\mathscr{G}$. Hence $\mathscr{G}$ is a subvariety of $\mathscr{S}$. Necessarily $\mathscr{G}$ is RS and, by the Lemma, $\mathscr{G}$ also has AP.

Following the notation of [11] we will denote by $\mathbf{R}(1,1)$ the semigroup on the set $\{0, a, 1\}$ where the element 1 is a left unit element and all other products are equal to 0 . We will denote by $\mathbf{L}(1,1)$ the semigroup on the same set with the opposite multiplication.

Lemma 3.3. Any residually small variety of semigroups containing $\mathbf{R}(1,1)$ or $\mathbf{L}(1,1)$ fails to have AP.

Proof. If $\mathscr{S}$ is residually small and contains $\mathbf{R}(1,1)$ then, for some $n, \mathscr{S}$ F $x^{n} \cdot y^{n} \cdot z \approx y^{n}: x^{n} \cdot z$ by [11]. However, no semigroup variety having AP and containing $\mathbf{R}(1,1)$ can satisfy this equation, as we now prove.

Assume that $\mathscr{S}$ has AP and contains $\mathbf{R}(1,1)$. Let $\mathbf{N}$ be the semigroup on $\{0, u, v\}$ where all products are equal to 0 . We have a diagram

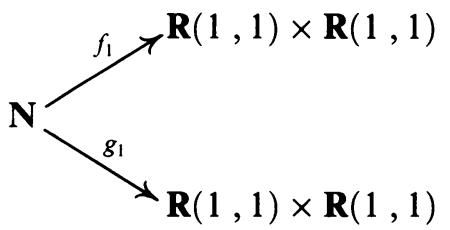

$$
\begin{array}{ll}
f_{1}(0)=(0,0), & g_{1}(0)=(0,0) \\
f_{1}(u)=(a, 0), & g_{1}(u)=(a, a) \\
f_{1}(v)=(a, a), & g_{1}(v)=(a, 0)
\end{array}
$$

Complete this diagram with a semigroup $\mathbf{P}$ and embeddings $f_{2}$ and $g_{2}$. $\mathbf{P}$ must contain elements $b=f_{2} \circ f_{1}(u), c=f_{2} \circ f_{1}(v), d=f_{2}((1,0))$, and $e=g_{2}((1,0))$. We see that $d \cdot b=f_{2}((1,0) \cdot(a, 0))=f_{2}((a, 0))=b=$ $f_{2}((1,0) \cdot(a, a))=d \cdot c$. Similarly that $e \cdot b=c=e \cdot c$. Hence, for all $n$ we see that $e^{n} \cdot\left(d^{n} \cdot b\right)=c \neq b=d^{n} \cdot\left(e^{n} \cdot b\right)$, so $\mathscr{S}$ is not residually small.

$\mathbf{L}(1,1)$ is handled in the same way.

Our notation for the next theorem is that $\mathbf{Z}_{2}$ is the two-element semilattice, $\mathbb{Z}_{2}$ is the two-element null semigroup (multiplication satisfies $x \cdot y \approx w \cdot z$ ), $\mathbf{R}_{2}$ is the two-element right-zero semigroup (multiplication satisfies $x \cdot y \approx y$ ) and $\mathbf{L}_{2}$ is the two-element left-zero semigroup. If $\mathbf{S}$ is a semigroup then $\mathbf{S}^{(0)}$ is the semigroup obtained from $\mathbf{S}$ by adding a new zero element. This notation follows [11].

Theorem 3.4. For a variety $\mathscr{S}$ of semigroups the following are equivalent:

(a) The subdirectly irreducible members of $\mathscr{S}$ are among the following semigroups:

$$
\mathbf{Z}_{2}, \boldsymbol{Z}_{2}, \mathbf{R}_{2}, \mathbf{L}_{2}, \mathbf{R}_{2}^{(0)}, \mathbf{L}_{2}^{(0)}
$$

along with finitely many semigroups of the form $\mathbf{G}$ or $\mathbf{G}^{(0)}$ where $\mathbf{G}$ is a cyclic group of prime-power order.

(b) $\mathscr{S}$ has the CEP.

(c) $\mathscr{S}$ satisfies AP $+\mathrm{RS}$.

(d) $\mathscr{S}$ is $R S, \mathbf{R}(1,1) \notin \mathscr{S}, \mathbf{L}(1,1) \notin \mathscr{S}$, and $\mathscr{S}$ contains no nonabelian groups. 
Proof. The fact that $(\mathrm{a}) \Rightarrow(\mathrm{b}) \Rightarrow(\mathrm{c})$ is given in [5]. To show that (c) implies (d), note that Lemma 3.3 proves that if $\mathscr{S}$ satisfies AP+RS then $\mathbf{R}(1,1)$ and $\mathbf{L}(1,1)$ are not elements of $\mathscr{S}$. Let $\mathscr{G} \subseteq \mathscr{S}$ be the subvariety of $\mathscr{S}$ consisting of groups. By Corollary 3.2 and the results of the last section, $\mathscr{G}$ also satisfies $\mathrm{AP}+\mathrm{RS}$ and hence $\mathscr{G} \vDash R$. If $\mathbf{G} \in \mathscr{G}$ is a nonabelian group, then we could choose $x \in[\mathbf{G}, \mathbf{G}] \backslash\left\{1_{\mathbf{G}}\right\}$ and set $\langle x\rangle=\mathbf{H}<\mathbf{G}$. Now, $\left[1_{\mathbf{G}}, 1_{\mathbf{G}}\right]_{\mathbf{H}}=1_{\mathbf{H}} \neq$ $0_{\mathbf{H}}=\left[1_{\mathbf{H}}, 1_{\mathbf{H}}\right]$, so $\mathscr{G}$ fails $R$. Consequently, $\mathscr{G}$ must be a variety of abelian groups.

That (a) follows from (d) is a consequence of the characterization of RS varieties of semigroups in terms of their subdirectly irreducible members given in [11].

A variety of monoids is closely related to the variety of semigroups that it generates. Because of this, it is easy to establish the following theorem.

Theorem 3.5. For a variety $\mathscr{M}$ of monoids the following are equivalent

(a) The subdirectly irreducible members of $\mathscr{M}$ are among the following monoids

$$
\mathbf{Z}_{2}
$$

along with finitely many monoids of the form $\mathbf{G}$ or $\mathbf{G}^{(0)}$ where $\mathbf{G}$ is a cyclic group of prime-power order.

(b) $\mathscr{M}$ has the CEP.

(c) $\mathscr{M}$ satisfies AP + RS.

(d) $\mathscr{M}$ is RS and contains no nonabelian groups.

Such a variety is a variety of strong semilattices of exponent- $n$ abelian groups.

\section{ReMARKS}

We make a few additional remarks about the implication $A P+R S \Rightarrow C E P$.

Suppose that $\mathscr{U}$ is a variety of unary algebras. If $\mathbf{B}$ is a subalgebra of $\mathbf{A}$, where $\mathbf{A} \in \mathscr{U}$, and if $\theta$ is a congruence on $\mathbf{B}$, then $\theta \cup 0_{\mathbf{A}}$ is a congruence on A extending $\theta$; hence $\mathscr{U}$ has the CEP. It is even easier to see that $\mathscr{U}$ has the AP. To complete the diagram

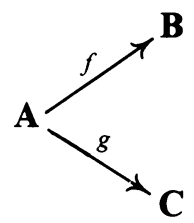

we only need to take the "union" of B and C (considering $f$ and $g$ to be inclusions). It is not hard to show that this algebra is a member of $\mathscr{U}$ if $\mathbf{B}$ and $\mathbf{C}$ are disjoint over $\mathbf{A}$. In [15] we find the theorem that any unary algebra may be embedded into a compact topological algebra (its Stone-Čech compactification). In particular, any unary algebra may be embedded into an 
equationally compact algebra. By W. Taylor's characterization of RS varieties [13], this is precisely what it means for $\mathscr{U}$ to be RS. Hence, unary varieties satisfy AP $+\mathrm{RS}+\mathrm{CEP}$. For varieties of algebras, this observation and the results of this paper subsume and extend the catalogue of information labelled "Table of Results" in [10] with respect to the implication $A P+R S \Rightarrow C E P$.

J. Berman has shown that any nonmodular variety that is generated by a twoelement algebra is either equivalent to the variety of semilattices (with possibly a nullary or constant unary operation added) or it is essentially unary [4]. From our results we conclude that two-element algebras generate varieties satisfying the implication AP+RS $\Rightarrow$ CEP. This remark is not deep, in fact it is not hard to use Berman's observation to give a proof that every two-element algebra generates a variety satisfying $A P+R S+C E P$ (i.e., an injectively complete variety).

The relationship between AP, RS and CEP can also be investigated for varieties generated by a finite algebra. The answer here is not known, but is is quite possible that more implications hold (see [8]).

\section{ACKNOWLEDGMENT}

I would like to thank R. McKenzie for introducing me to this problem and also for his encouragement and help. The idea for Lemma 3.3 is his.

\section{REFERENCES}

1. C. Bergman, On the relationship of $A P, R S$ and $C E P$ in congruence modular varieties, Algebra Universalis 22 (1986), 164-171.

2. $\ldots$, Another consequence of AP in residually small, congruence modular varieties, Houston $\mathbf{J}$. Math. (to appear).

3. C. Bergman and R. McKenzie, On the relationship of $A P, R S$ and $C E P$ in congruence modular varieties. II, preprint.

4. J. Berman, A proof of Lyndon's finite basis result, preprint.

5. B. Biró, E. Kiss, and P. Pálfy, On the congruence extension property, Universal Algebra, pp. 129-151 (Proc. Coll. Math. Soc. J. Bolyai, Esztergom, 1977).

6. R. Freese and R. McKenzie, Comutator Theory for Congruence Modular Varieties, Lecture Notes in Math., vol. 125, Cambridge, 1987.

7. G. Grätzer, General lattice theory, Birkhäuser Verlag, Basel-Stuttgart, 1978.

8. K. A. Kearnes, Finite algebras that generate an injectively complete variety, preprint.

9. E. Kiss, Injectivity and related concepts in moduular varieties. I-II, Bull. Austral. Math. Soc. 32 (1985), 35-53.

10. E. Kiss, L. Márki, P. Pröhle and W. Tholen, Categorical algebraic properties. A compendium on amalgamation, congruence extension, epimorphisms, residual smallness, and injectivity, Studia Sci. Math. Hungar. 18 (1983), 79-141.

11. R. McKenzie, Residually small varieties of semigroups, Algebra Universalis 13 (1981), 171201.

12. R. McKenzie, G. McNulty and W. Taylor, Algebras, lattices, varieties, Wadsworth Inc. and Books/Cole Publishing Co., 1987. 
13. W. Taylor, Residually small varieties, Algebra Universalis 2 (1972), 33-53.

14. __ Equational logic, Houston J. Math., 1979, 83 pp.

15. B. Weglorz, Remarks on compactifications of abstract algebras (abstract), Colloq. Math. 14 (1966), 372.

Department of Mathematics, University of California, Berkeley, California 94720

Current address: Department of Mathematics, The University of Hawaii at Manoa, Honolulu, HI 96822 